\title{
Solar activity: periodicities beyond 11 years are consistent with random forcing
}

\author{
R. H. Cameron and M. Schüssler
}

\begin{abstract}
Max-Planck-Institut für Sonnensystemforschung Justus-von-Liebig-Weg 3, 37077 Göttingen, Germany e-mail: cameron@mps.mpg.de
\end{abstract}

Received 16 February 2019 / Accepted 13 March 2019

\begin{abstract}
Power spectra of solar activity based on historical records of sunspot numbers and on cosmogenic isotopes show peaks with enhanced power apart from the dominant 11-year solar cycle, such as the 90-year Gleissberg cycle or the 210-year de Vries cycle. In a previous paper we have shown that the overall shape of the power spectrum is well represented by the results of the generic normal form model for a noisy and weakly nonlinear limit cycle, with parameters all determined by observations. Using this model as a null case, we show here that all local peaks with enhanced power, apart from the 11-year band, are consistent with realization noise. Even a $3 \sigma$ peak is expected to occur with a probability of about 0.25 at least once among the 216 period bins resolved by the cosmogenic isotope data. This casts doubt upon interpretations of such peaks in terms of intrinsic periodicities of the solar dynamo process.
\end{abstract}

Key words. Sun: general - Sun: magnetic fields

\section{Introduction}

Solar activity has been directly observed in terms of sunspot numbers since about 400 years, while its level at earlier times can be inferred from the concentrations of the cosmogenic isotopes ${ }^{14} \mathrm{C}$ in tree rings and ${ }^{10} \mathrm{Be}$ in polar ice over the last $\sim 10000$ years. In addition to the 11-year Schwabe cycle, these records show modulation of the activity level on longer time scales. Power spectra show signal at all periods, together with a number of peaks of locally enhanced power. Peaks around 90 years (generally called the Gleissberg cycle), 210 years (de Vries cycle), and perhaps 2400 years (Hallstatt cycle) of presumably solar origin (McCracken et al. 2013) have been discussed in the literature (see Usoskin 2017, and references therein). Although it is often assumed that these periodicities are intrinsic to the solar dynamo process (e.g., Beer et al. 2018), in the absence of a proper null case it has so far not been tested whether these power peaks are consistent with noise due to stochastic random processes affecting the dynamo. Even if such peaks are formally significant (say, at the $3 \sigma$ level), this does not necessarily exclude that they originate from random noise. Here we propose an appropriate null case and test.

In Cameron \& Schüssler (2017a) we show that the solar dynamo can be reasonably described by a weakly nonlinear limit cycle affected by random noise. Such a model is favored by stellar observations suggesting that the Sun is near the critical rotation rate for which dynamo action sets in (van Saders et al. 2016; Metcalfe et al. 2016; Metcalfe \& van Saders 2017) and by solar observations of the scatter of active region tilt angles (Jiang et al. 2014), which are a basic ingredient of BabcockLeighton-type models of the solar dynamo (Charbonneau 2010; Cameron \& Schüssler 2017b). This approach leads to a normalform model for a noisy system near a Hopf bifurcation, which is generic in the sense that it does not depend on the detailed properties of the system, such as its specific kind of nonlinearity. The parameters of this model are all well constrained by solar observations. Random time series resulting from the model show power spectra consistent with the overall shape of the power spectrum of solar activity derived from the sunspot record and cosmogenic isotopes, including the occurrence and statistics of grand minima, in other words, extended periods of very low activity (Cameron \& Schüssler 2017a).

These results suggest that the noisy normal-form model (the NNF model) provides an adequate null case for evaluating the significance of the various discrete periodicities (e.g., the Gleissberg, de Vries, and Hallstatt cycles) that have been detected in the power spectra based on cosmogenic isotope records. In this paper, we consider whether such peaks are consistent with realization noise of the generic normal-form model.

\section{Data and model}

We used the reconstructed record of solar activity based on cosmogenic isotopes provided by Usoskin et al. (2016). To exclude a dependence on the specific reconstruction we also employed the data of Steinhilber et al. (2012) and found similar results. We compared these empirical data with realizations of the NNF model (Cameron \& Schüssler 2017a) given, in terms of a stochastic differential equation, by

$\mathrm{d} X=\left(\beta+i \omega_{0}-\left(\gamma_{r}+i \gamma_{i}\right)|X|^{2}\right) X \mathrm{~d} t+\sigma X \mathrm{~d} W_{c}$,

where $X$ is a complex variable whose real and imaginary parts represent, respectively, the poloidal and toroidal magnetic field components. As a measure of the activity level, we take $|\operatorname{Re}(X)|$. $\omega_{0}=2 \pi / 22 \mathrm{yr}^{-1}$ is the linear frequency of the magnetic cycle and the linear growth rate, $\beta=1 / 50 \mathrm{yr}^{-1}$, is based on the recovery time from the Maunder minimum. The parameters $\gamma_{r}$ and $\gamma_{i}$ determine the cycle amplitude, $|X|=\sqrt{\beta / \gamma_{r}}$, and the nonlinear cycle frequency, $\omega=\omega_{0}-\gamma_{i} \beta / \gamma_{r}$. We have assumed the cycle frequency to be unaffected by the (weak) nonlinearity, i.e., $\gamma_{i}=0$, and scale the cycle amplitude in terms of the average group 

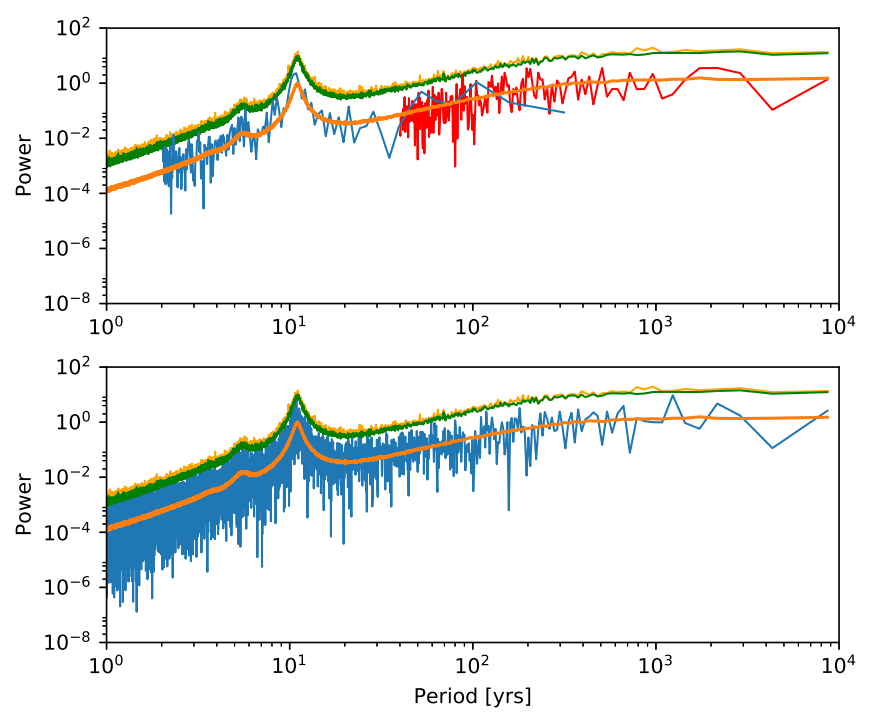

Fig. 1. Observed and modeled power spectra of solar activity. Upper panel: spectra based on the historical record of sunspot numbers (blue curve) and on the reconstruction from cosmogenic isotope data (Usoskin et al. 2016, red curve). Lower panel: spectrum obtained from one realization of the noisy normal form model (NNF model Cameron \& Schüssler 2017a, blue curve). In both panels, the orange curve gives the median from 1000 realizations of the model, the green curve shows the corresponding $99.865 \%$ ( $3 \sigma$ ) upper percentile, and the yellow curve represents the maximum of the 1000 realizations.

sunspot number since 1700 , yielding $\gamma_{r}=\beta / 64^{2}$. For the noise term, $W_{c}$, we consider a complex Wiener process with Gaussian distributed increments and an amplitude of $\sigma=0.4 / \sqrt{11} \mathrm{yr}^{-1 / 2}$, estimated from the variability of the observed cycle amplitudes. For our analysis, we computed a set of 1000 different realizations of the NNF model, each covering 10000 years. For the numerical solution, we used the Euler-Maruyama method with a timestep of 1 day. Further details can be found in Cameron \& Schüssler (2017a).

\section{Results}

\subsection{Significance of single peaks}

Estimating the significance of peaks in a power spectrum requires a null case. Since the overall shape of the empirical spectrum based on cosmogenic isotopes is well represented by the NNF model (Cameron \& Schüssler 2017a), we propose this model as a physics-based null case instead of an ad-hoc choice such as red or white noise. For the NNF model we can determine the standard deviation from the median spectrum (as well as the significance of local peaks) by considering a sufficient number of random realizations. Figure 1 shows power spectra from the record of solar activity (observed sunspot numbers ${ }^{1}$ and inferred from cosmogenic isotope data) in the upper panel and from one realization of the NNF model in the lower panel. In both panels, the orange curve gives the median of the 1000 model realizations, the green curve shows the (upper) $99.865 \%$ percentile level from the set of model realizations (corresponding to the $3 \sigma$ level), and the yellow curve displays the maximum power for each frequency point of the 1000 realizations. The near coincidence of the green and yellow curves indicates that, as expected

\footnotetext{
1 Version 2 of the yearly sunspot number: http://www.sidc.be/ silso/DATA/SN_y_tot_V2.0.txt
}

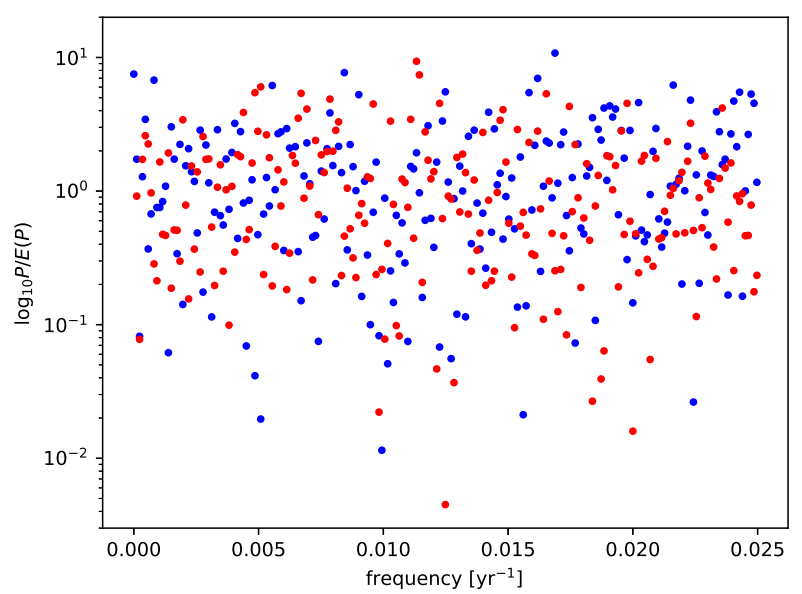

Fig. 2. Ratio of the power in a frequency bin divided by the median power of the NNF model realizations at that frequency. The blue points correspond one realization of the noisy normal form model, the red points to the reconstruction from cosmogenic isotopes (cf. Fig. 1).

on statistical grounds, for 1000 realizations of a random process at least one peak above the $3 \sigma$ level in one of these realizations is expected for any given frequency. Considering the 216 periods over 40 years that are resolved by the cosmogenic isotope data, the probability, $p$, of finding at least one peak above the $3 \sigma$ level in one realization of the random model (with the same period resolution) is given by

$p=1-0.99865^{216}=0.253$,

so that we expect such a peak in about a quarter of the realiations. Therefore, the appearance of an outlier $3 \sigma$ peak such as that corresponding to the 88-year Gleissberg period in the Usoskin et al. (2016) data is quite consistent with the random model containing no intrinsic frequencies apart from the 11-year cycle. We note that the probability of 0.253 for a $3 \sigma$ peak is independent of the null case used for determining $\sigma$ : it is based on a $\sim 0.00135$ probability of finding a $3 \sigma$ peak at any given resolved frequency bin, and having 216 resolved frequency bins.

As a further comparison of the empirical and model power spectra, we consider the scatter with respect to the expectation value (median of the 1000 NNF model realizations) shown in Fig. 2. Application of the Kolmogorov-Smirnov test shows that the distributions for the empirical data and for the NNF model (red and blue dots, respectively) are consistent with each other at the $2 \sigma(95 \%)$ level. This is further illustrated by Fig. 3, which shows probability distribution functions (PDF) of the ratio between the power at a given frequency and the median of the model realizations, considering all periodicities above 40 years. The consistency of the scatter in the empirical power spectrum with the NNF model demonstrates that the model captures the noise statistics of the empirical data quite well.

Another property that sheds light upon the nature of peaks in the power spectra is their sharpness. The $3 \sigma$ peaks appearing in the NNF model are usually quite narrow (at most a few resolution elements in frequency). This results from the fact that the random noise in the model is uncorrelated in frequency space. On the other hand, an intrinsic periodicity of the dynamo process is expected to yield a broader peak (such as the 11-year band) since the noise effectively leads to a finite coherence time of the dynamo modes. Figure 4 demonstrates that the peaks corresponding to the observed Gleissberg and de Vries periodicities indeed are quite sharp, similar to those resulting from the NNF model. 


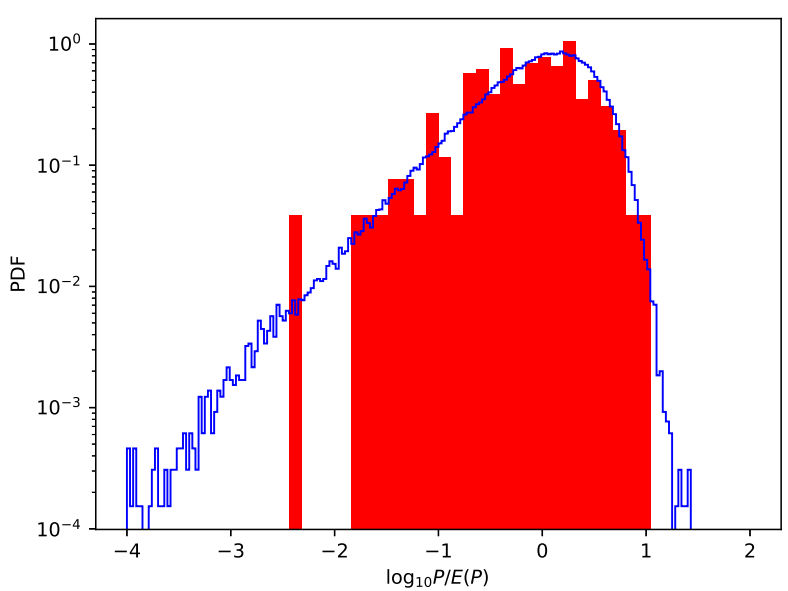

Fig. 3. Probability distribution function of the ratio of the power in a frequency divided by the median power of the NNF model realizations at that frequency. The blue curve is the expectation value based on 1000 realizations of the model, the red bars correspond to the reconstruction from cosmogenic isotopes.
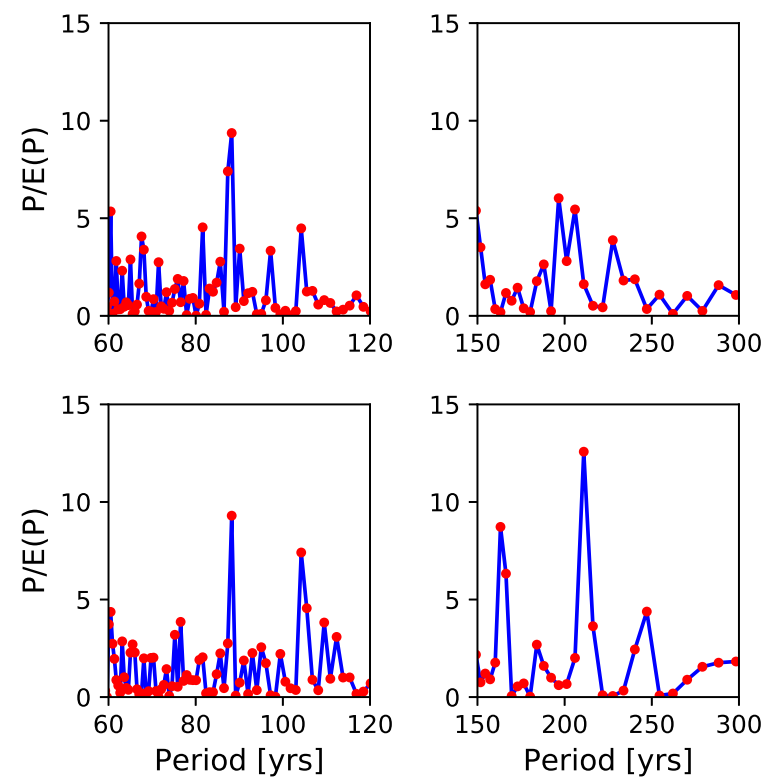

Fig. 4. Segments from power spectra around the Gleissberg (left panels) and the de Vries (right panels) periodicities. Shown is the ratio of power to the expectation value (median of 1000 realizations of the NNF model). Spectra from reconstruction based upon cosmic isotopes by (upper panels) are compared with those from selected realizations of the NNF model with $3 \sigma$ peaks in the same range of periods (lower panels). The red dots emphasize the frequency resolution. The peaks from the empirical data are similarly sharp as those from the model, indicating the random origin of the former.

\subsection{Analyzing subseries}

It has been argued that the appearance of certain periods during different stretches of time throughout the interval covered by the cosmogenic isotope data would provide evidence against a random origin (e.g., Svalgaard 2018). This can easily be tested using the NNF model as a null case.

For 1000 realizations of the NNF model covering 8650 years each, we applied the Lomb-Scargle periodogram method and selected the 216 realizations with the highest (3-sigma) peaks. For the thus selected realizations and peaks, we determined the

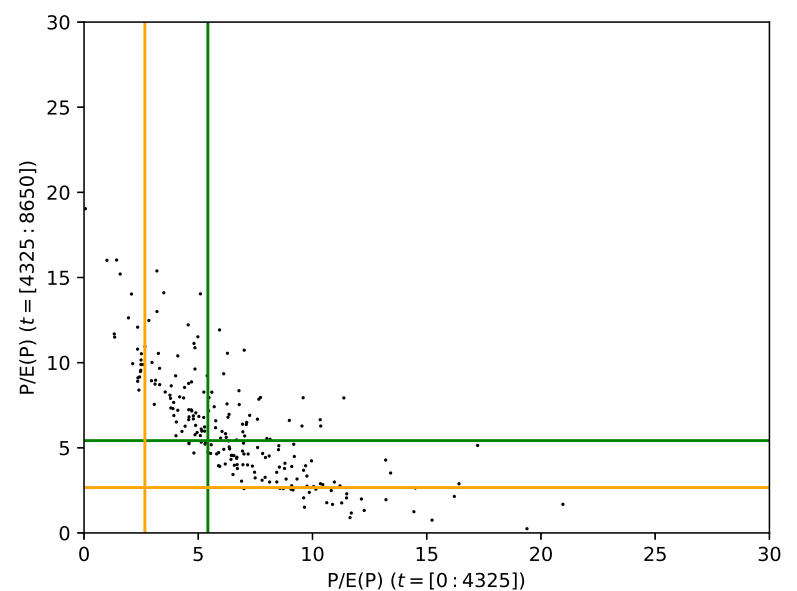

Fig. 5. Period analysis of subseries from realizations of the NNF model. Each point in the diagram corresponds to one of the 216 highest peaks with periods above 40 years in the normalized power spectra (power divided by the expectation value given by the median) from 1000 realizations. They are plotted according to the normalized power at the same period in the first half (horizontal axis) and in the second half (vertical axis) of the 10000 years full series. The distribution of the points show that significant power in the full series in most cases corresponds to high power in both subseries. $84 \%$ of all points in the power spectrum from the two subseries lie beneath (to the left of) the orange horizontal (vertical) line and $97.7 \%$ lie beneath (to the left of) the green lines.

spectral power at the frequencies corresponding to these peaks when the first and second halves of the corresponding time series were analyzed separately. The values of the normalized power thus obtained for the first and second halves of the random series are plotted against each other in Fig. 5. The resulting distribution shows that in most cases of a significant spectral peak in the full series, there is significant power as well for both half series. Since all peaks in the NNF model (without intrinsic periodicities apart from the 11-year cycle) are due to stochastic noise, this demonstrates that the consistent appearance of high peaks in both subseries does not per se provide evidence for a nonrandom (intrinsic) nature of such peaks. It is clear that there is always a better chance for a significant peak in the full series if it is already strong in both half series.

\subsection{Bandpass filtering}

Bandpass filtering of the power spectrum in the range of the Gleissberg and de Vries periods and back transformation into the time domain has been used, for example, by Beer et al. (2018) in an attempt to elucidate the nature of the periodicities found in the empirical data. However, applying the same procedure to realizations of the NNF model shows that bandpass filtering does not provide any evidence for the intrinsic nature of these periodicities.

We consider the two realizations of the NNF model that show peaks in the range of the Gleissberg and de Vries periodicities (cf. Fig. 4). The time evolution of the sunspot number (SSN) for these realizations is shown in Fig. 6, together with the corresponding record of the reconstructed sunspot number based on cosmogenic isotopes (Usoskin et al. 2016). Periods below 40 years were filtered out in all three time series.

Figures 7 and 8 show, respectively, the results of passband filtering in the ranges 75-100 yr (Gleissberg) and 180-230 yr (de Vries) in comparison with the corresponding pattern obtained by applying the same filtering to the empirical record. The patterns 

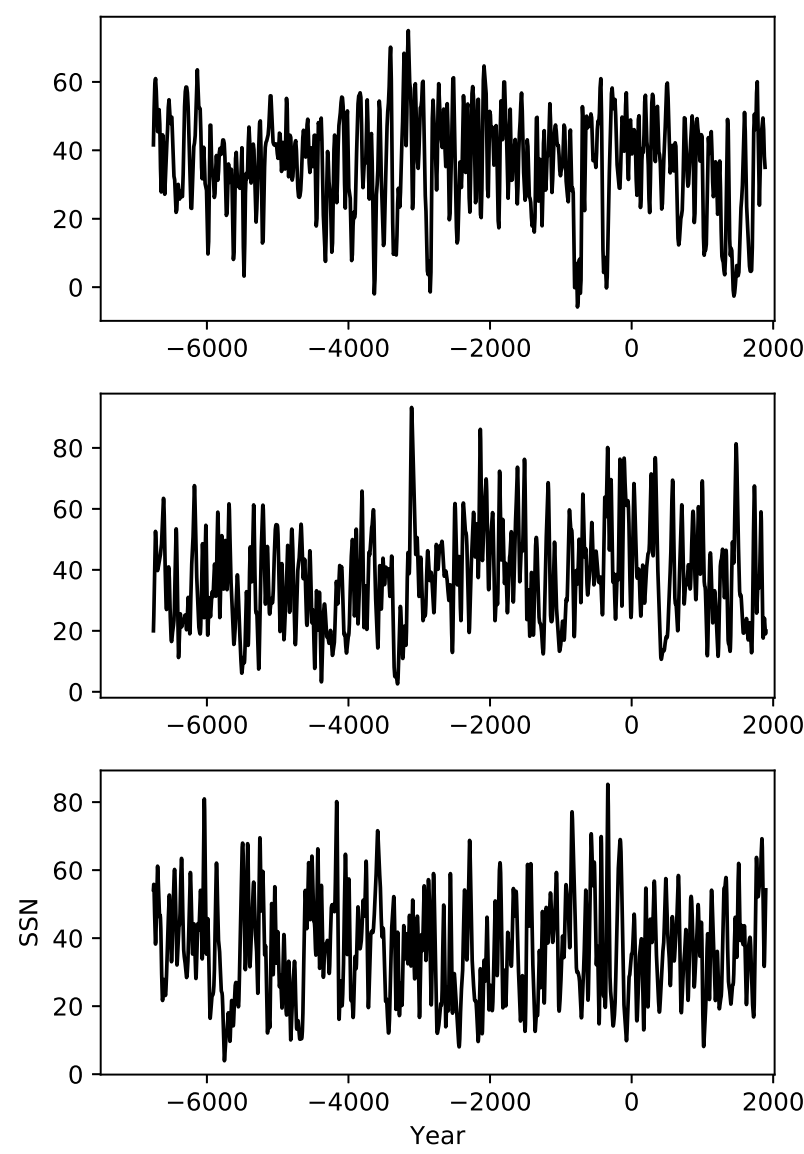

Fig. 6. Long-term activity records. Top panel: reconstructed sunspot number based on cosmogenic isotopes (Usoskin et al. 2016). Middle panel: realization of the noisy normal form model with a $3 \sigma$ peak in the range of the Geissberg period. Bottom panel: realization of the noisy normal form model with a $3 \sigma$ peak in the range of the de Vries period (cf. Fig. 4).
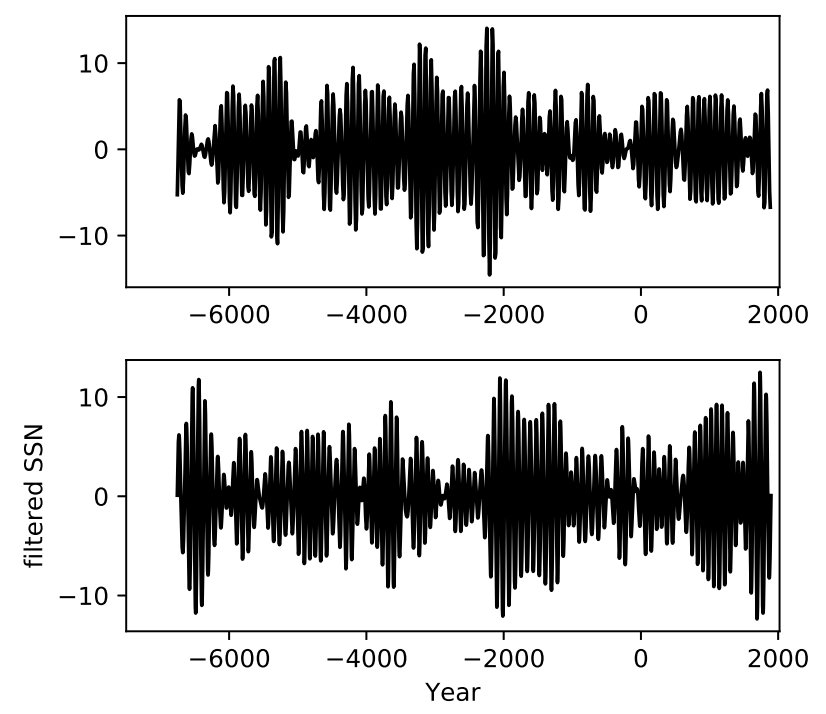

Fig. 7. Passband filtered time evolution of the sunspot number in the period range between $75 \mathrm{yr}$ and $100 \mathrm{yr}$ around the Gleissberg period. The result from the empirical record reconstructed from cosmogenic isotopes (upper panel) are shown in comparison to that from a realization of the noisy normal form model (lower panel).
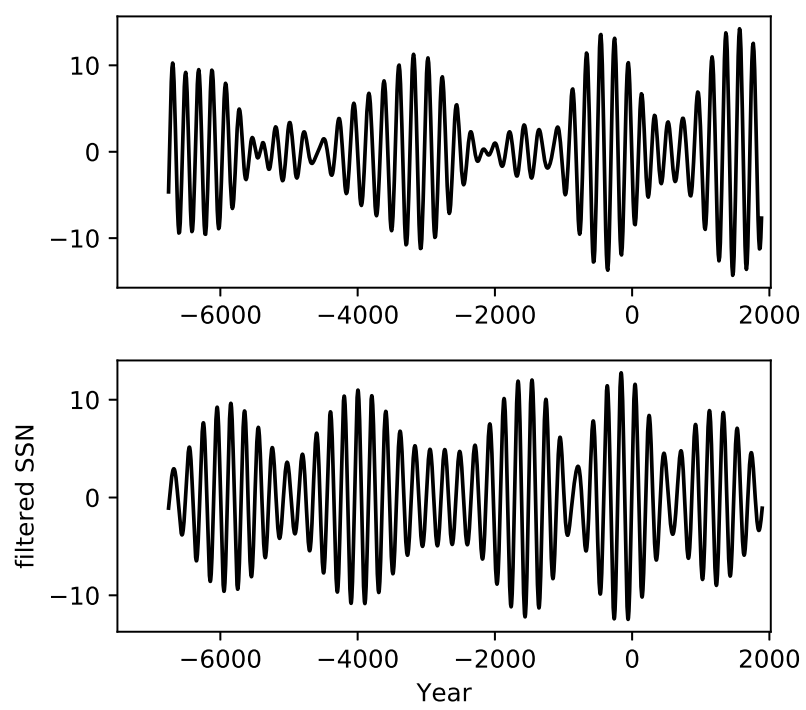

Fig. 8. Same as Fig. 7 with passband filtering in the range between $180 \mathrm{yr}$ and $230 \mathrm{yr}$ around the de Vries period.
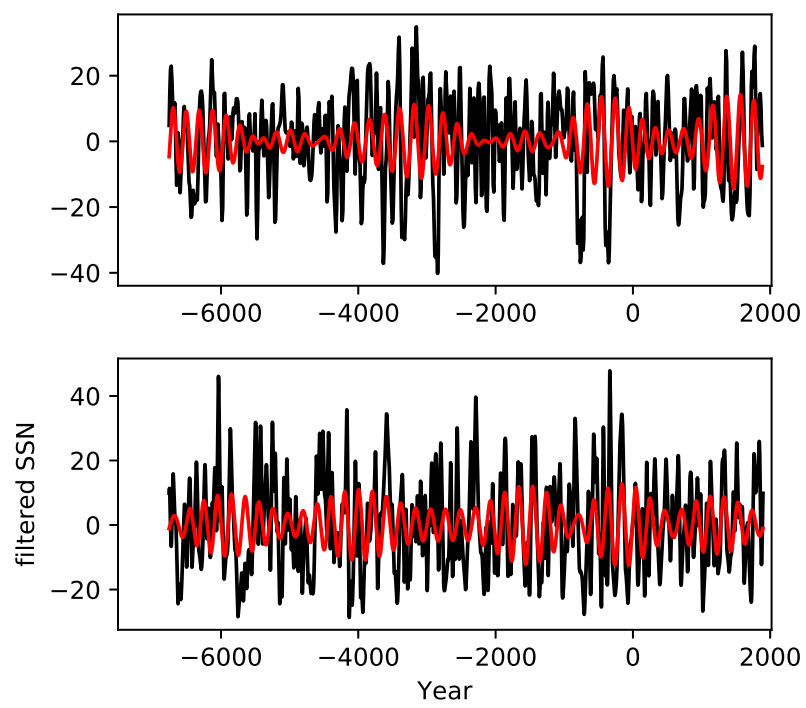

Fig. 9. High-pass filtered records of the empirical (upper panel) and modeled sunspot numbers (lower panel). Overplotted in red are the corresponding bandpass-filtered records in the range of the de Vries period (cf. Fig. 8).

from the NNF model and from the real data are qualitatively similar, suggesting that the corresponding frequency (Gleissberg and de Vries) seen in the cosmogenic isotope record is consistent with a random process.

Finally, Fig. 9 shows the empirical record after bandpass filtering in the range 40-1000 years (black curve) and 180230 years (around the de Vries perio, red curve), together with the result after applying the same procedures to the realization of the NNF model. This figure can be compared to the analogous Fig. 5 of Beer et al. (2018). The extrema of the filtered de Vries periodicities tend to align with some of the extrema (grand minima and grand maxima) of the original series, giving the impression of a periodic appearance of such extrema. However, this is also the case in the NNF series with a random excitation of a periodicity in the same range. 
Since bandpass filtering of the NNF realizations leads to qualitatively similar results as applying the same procedure to the empirical data, we conclude that such filtering does not provide evidence for the intrinsic nature of the related periodicities.

\section{Conclusion}

Our analysis shows that the fluctuations in the power spectrum of the sunspot numbers reconstructed from cosmogenic isotopes are consistent with a weakly nonlinear and noisy limit cycle with no intrinsic periodicities except that of the basic 11/22year cycle. Such a mode of operation of the solar dynamo is suggested by solar and stellar observations and can be faithfully described by a generic noisy normal form model with parameters taken from observations (Cameron \& Schüssler 2017b). Seemingly significant periodicities such as the $\sim 90$-year Gleissberg and the $\sim 210$-year de Vries "cycles" are expected to occur in random realizations as a result of the stochastic noise in the dynamo excitation. This conclusion is further strengthened by the sharpness of the corresponding peaks in the power spectrum, indicating a random origin.

Of course, our analysis cannot per se exclude that these periodicities may be intrinsic after all, but we have shown that, so far, this notion is not supported by the data, which are consistent with the NNF null case. The interpretation of such periodicities in terms of dynamo theory (e.g., Beer et al. 2018) should therefore be considered with due caution.

Acknowledgements. I. G. Usoskin kindly provided the sunspot number reconstruction from the cosmogenic isotope record presented in Usoskin et al. (2016).

\section{References}

Beer, J., Tobias, S. M., \& Weiss, N. O. 2018, MNRAS, 473, 1596

Cameron, R. H., \& Schüssler, M. 2017a, ApJ, 843, 111

Cameron, R. H., \& Schüssler, M. 2017b, A\&A, 599, A52

Charbonneau, P. 2010, Liv. Rev. Sol. Phys., 7, 3

Jiang, J., Cameron, R. H., \& Schüssler, M. 2014, ApJ, 791, 5

McCracken, K. G., Beer, J., Steinhilber, F., \& Abreu, J. 2013, Sol. Phys., 286 609

Metcalfe, T. S., \& van Saders, J. 2017, Sol. Phys., 292, 126

Metcalfe, T. S., Egeland, R., \& van Saders, J. 2016, ApJ, 826, L2

Steinhilber, F., Abreu, J. A., Beer, J., et al. 2012, Proc. Natl. Acad. Sci., 109, 5967

Svalgaard, L. 2018, ArXiv e-prints [arXiv:1810. 11952]

Usoskin, I. G. 2017, Liv. Rev. Sol. Phys., 14, 3

Usoskin, I. G., Gallet, Y., Lopes, F., Kovaltsov, G. A., \& Hulot, G. 2016, A\&A, 587, A 150

van Saders, J. L., Ceillier, T., Metcalfe, T. S., et al. 2016, Nature, 529, 181 